\title{
1 Hormetic effect of ionic liquid 1-ethyl-3-methylimidazolium acetate on
}

2 bacteria

3 Y. V. Nancharaiah ${ }^{1, *}$ and A. J. Francis ${ }^{2,3}$

$4 \quad{ }^{1}$ Biofouling and Biofilm Processes Section, Water and Steam Chemistry Division, Bhabha

5 Atomic Research Centre, Kalpakkam - 603102, Tamil Nadu, India.

6 2 Environmental Sciences Department, Brookhaven National Laboratory, Upton, NY 11973, 7 USA.

$8 \quad{ }^{3}$ Division of Advanced Nuclear Engineering, POSTECH, Pohang, Korea.

9 *Corresponding author: Y.V. Nancharaiah, Email: venkatany@gmail.com, yvn@igcar.gov.in, 10 Phone: +9144 27480203, Fax: +9144 27480097

11 Abstract

12 The biological effect of ionic liquids (ILs) is one of the highly debated topics as they are being

13 contemplated for various industrial applications. 1-ethyl-3-methylimidazolium acetate

14 ([EMIM][Ac]) showed remarkable hormesis on anaerobic Clostridium sp. and aerobic

Pseudomonas putida. Bacterial growth was stimulated at up to $2.5 \mathrm{~g} \mathrm{~L}^{-1}$ and inhibited at $>2.5 \mathrm{~g}$ $\mathrm{L}^{-1}$ of [EMIM][Ac]. The growth of Clostridium sp. and $P$. putida were higher by 0.4 and 4 -fold respectively, in the presence of $0.5 \mathrm{~g} \mathrm{~L}^{-1}$ [EMIM][Ac]. Assessment of the effect of [EMIM][Ac] under different growth conditions showed that the hormesis of [EMIM][Ac] was mediated via regulation of medium $\mathrm{pH}$. Hormetic effect of [EMIM][Ac] was evident only in medium with poor buffering capacity and in the presence of a fermentable substrate as the carbon source. The hormetic effect of [EMIM][Ac] on bacterial growth is most likely associated with the buffering capacity of acetate anion. These observations have implications in ILs toxicity studies and ecological risk assessment.

Keywords: 1-ethyl-3-methylimidazolium acetate; Biomass pretreatment; Clostridium sp.; hormesis; imidazolium ionic liquids; Pseudomonas putida. 


\section{Introduction}

Ionic liquids (ILs) are novel class of organic salts with low melting points $\left(<100{ }^{\circ} \mathrm{C}\right)$, increasingly considered as green replacements for volatile organic compounds (Passos et al., 2014; Patel et al., 2012). Ionic liquids are typically made up of two components, a bulky organic cation (i.e. N,N'-dialkylimidazolium, N-alkylpyridinium, alkylammonium, alkylphosphonium, alkylsulphonium and triazolium) and an organic or inorganic anion (i.e. halides, tetrafluoroborate, hexafluorophosphate, alkylphosphates, acetate) (Bubalo et al., 2014). These compounds have been in the spotlight of scientific and industrial community as novel green solvents for replacement of conventional volatile solvents (Bubalo et al., 2014). Ionic liquids are attractive due to their low vapor pressure, non-flammability, and high thermal stability. Importantly, ionic liquids offer unprecedented flexibility in designing several classes of compounds with novel physical and chemical properties by means of tuning cation and anion structure (Earle and Seddon, 2000). Ionic liquids are extensively studied for applications in organic synthesis, separation technology, biocatalysis, corrosion inhibitors, biomass pretreatment and in use as corrosion inhibitors and antimicrobials (Brand et al., 2013; Pham et al., 2010; Plechkova and Seddon 2008; Nancharaiah et al., 2012).

Lignocellulosic materials (i.e. wood, agricultural or forest residues) are most abundant on our planet and available at a much lower cost than starch and sucrose based materials for production of biofuels (Brandt et al., 2013). However, major obstacle in using the lignocellulosic materials is the non availability of cost-effective pretreatment technologies for hydrolysis and deconstruction to readily fermentable products (Datta et al., 2010). Ionic liquid based pretreatment methods show promise for cellulose dissolution and biomass deconstruction (Brand 
et al., 2013; Zavrel et al., 2009). Ionic liquids with chloride, acetate, and phosphate anions showed good cellulose dissolution capacities (Vitz et al., 2009). The cellulose dissolving abilities of 1-ethyl-3-methylimidazolium acetate ([EMIM][Ac]), 1-ethyl-3-methylimidazolium dimethylphosphate ([EMIM][DEP]) and 1-methyl-3-methylimidazolium dimethylphosphate ([MMIM] $[\mathrm{DMP}])$ were reported to be in the range of $8 \%, 10 \%$ and $12-14 \%(\mathrm{w} / \mathrm{v})$ respectively (Vitz et al., 2009). Moreover, the pretreatment methods should neither introduce nor generate compounds that would negatively impact the downstream processes such as fermentation. Interestingly, the growth and fermentative metabolism of Clostridium sp. was not inhibited by 1ethyl-3-methyl imidazolium and 1-methyl-3-methyl imidazolium ionic liquids with anions such as acetate, dimethylphosphate or diethylphosphate up to $2.5 \mathrm{~g} \mathrm{~L}^{-1}$ (Nancharaiah and Francis, 2011).

High volume production and wide applications of ionic liquids could lead to pollution of aquatic environments due to water solubility of ILs (Zhang et al., 2011). Many studies have shown that ILs are persistent in the environment and exhibit toxicity towards prokaryotic and eukaryotic organisms (Bubalo et al., 2014; Pham et al., 2010). However, the toxicity of IL is dependent on cation, alkyl chain length of substituent of cation, and anion. Recently, hormesis was observed in case of certain ionic liquids, particularly those with short alkyl chains (Ge et al., 2010; Nancharaiah and Francis 2011; Wang et al., 2011; Zhang et al., 2013). Hormesis was originally applied to describe the effect of low doses of ionizing radiation, but now it is generally used to describe biphasic dose-response of biological systems to environmental conditions or stress (Davies et al., 2006). In toxicology, hormesis is defined as a biphasic dose-response phenomenon primarily characterized by stimulation of biological response at lower concentrations while inhibition at higher concentrations. The hormetic response of ionic liquids 
72 is a poorly understood phenomenon and the chemical and biochemical mechanisms are

73 unknown. Among the three ionic liquids (i.e. [EMIM][Ac], [EMIM][DEP], [MMIM][DMP]),

74 tested for their influence on the growth and fermentative metabolism of Clostridium sp. BC1,

75 only [EMIM][Ac] showed hormetic effect. Consequently, the aim of the present study was to

76 investigate the mode of action of hormesis by determining the hormetic effect of [EMIM][Ac] on

77 anaerobic Gram +ve and Gram -ve bacteria under different growth conditions.

\section{2. Materials and methods}

792.1 Ionic liquids

80

81 82 received.

83

84

85

86

87

88

89

The structures of ILs [EMIM][Ac], [EMIM][DEP] and [MMIM][DMP] used in the present study are shown in Table 1. All the ionic liquids were obtained from Sigma-Aldrich and used as

\subsection{Ionic liquid stock solutions}

Stock solutions of ionic liquids were prepared in de-ionized water as described earlier (Nancharaiah and Francis, 2011). The ionic liquid solutions were sterilized by filtering through $0.22 \mu \mathrm{m}$ Millex filter. The ionic liquid solutions were transferred to serum bottles, closed with butyl rubber stoppers, aluminum crimp sealed and deoxygenated by purging with ultra high purity (UHP) $\mathrm{N}_{2}$ gas. The ionic liquid stock solutions were stored at room temperature.

\subsection{Bacterial cultures and growth conditions}


Clostridium sp. BC1 (ATCC 53464), gram-positive, anaerobic, fermentative bacterium, is phylogenetically closely related to $C$. pasteurianum. It was grown in mineral salts medium in serum bottles as described earlier (Nancharaiah and Francis, 2011). The mineral salts medium contained the following: glucose, $10.0 \mathrm{~g}$; $\mathrm{NH}_{4} \mathrm{Cl}, 0.5 \mathrm{~g}$; glycerol phosphate, $0.3 \mathrm{~g}$; $\mathrm{MgSO}_{4} .7 \mathrm{H}_{2} \mathrm{O}$, $0.2 \mathrm{~g} ; \mathrm{CaCl}_{2} .2 \mathrm{H}_{2} \mathrm{O}, 0.5 \mathrm{~g} ; \mathrm{FeSO}_{4} .7 \mathrm{H}_{2} \mathrm{O}, 0.005 \mathrm{~g}$; peptone, $0.1 \mathrm{~g}$; yeast extract, $0.1 \mathrm{~g}$; deionized water, $1 \mathrm{~L} ; \mathrm{pH}, 6.8$. The medium contained glycerol phosphate as the $\mathrm{P}$ source. The medium was pre-reduced by boiling for 10 min while purging with UHP nitrogen gas. The medium was dispensed as $40 \mathrm{~mL}$ aliquots into $60 \mathrm{~mL}$ serum bottles in an anaerobic chamber (Coy Laboratory products, USA). The serum bottles containing media were fitted with butyl rubber stoppers, crimp sealed with aluminum caps and autoclaved. The culture was maintained by repeated subculturing in serum bottles by inoculating autoclaved MS medium with $1 \mathrm{~mL}$ of log phase culture. The serum bottles were incubated at $26^{\circ} \mathrm{C}$.

\section{Pseudomonas putida TUM-PP12 (Nancharaiah et al., 2003, 2008), a gram-negative} bacterium, was maintained in Luria Bertani agar (Difco, USA) plates supplemented with $50 \mu \mathrm{g}$ $\mathrm{mL}^{-1}$ kanamycin under aerobic conditions. For liquid cultures, $P$. putida was routinely grown in $250 \mathrm{~mL}$ Erlenmeyer flasks containing $100 \mathrm{~mL}$ sterile mineral salts medium by inoculating with $\log$ phase culture. The culture flasks were incubated at $30^{\circ} \mathrm{C}$ in an orbital shaker set at $100 \mathrm{rpm}$.

\subsection{Effect of ionic liquids on Clostridium sp. and P. putida}

To determine the effect of ILs on Clostridium sp. different concentrations $\left(0.5\right.$ to $10 \mathrm{~g} \mathrm{~L}^{-1}$ w/v) of ILs were added to serum bottles containing sterile mineral salts medium, Sterile mineral salts medium without ionic liquids was used as control. The serum bottles with and without 
111 ionic liquids in mineral salts medium were inoculated with $1 \mathrm{~mL}$ of 24 h-old Clostridium sp.

112 culture (OD 0.4). The serum bottles were incubated at $26^{\circ} \mathrm{C}$. At periodic time intervals, total gas

113 production was measured. After measuring the total gas production, a $4 \mathrm{~mL}$ of the culture sample

114 was removed with a syringe for monitoring growth and medium $\mathrm{pH}$.

115 To determine the effect of ILs on P. putida, Erlenmeyer flasks ( $250 \mathrm{~mL}$ volume) containing

$116100 \mathrm{~mL}$ of sterile mineral salts medium with and without [emim]Ac were inoculated with $1 \mathrm{~mL}$

117 of $24 \mathrm{~h}$ old culture of $P$. putida. The flasks were incubated at $30{ }^{\circ} \mathrm{C}$ in an orbital shaker set at 100

$118 \mathrm{rpm}$. Liquid samples were collected at regular time intervals for measuring growth and $\mathrm{pH}$. The

119 effect of [EMIM][Ac] on the growth of $P$. putida was also determined in MS medium

120 supplemented with acetate as sole carbon source and tryptic soy broth (Difco, USA) under the

121 similar experimental conditions.

$122 \quad 2.5$ Effect of ionic liquids on growth in phosphate-buffered mineral salts medium

123 In order to understand the hormetic effect of [EMIM][Ac], the growth of Clostridium sp. and 124 P. putida was determined in phosphate buffered mineral salts (PMS) medium (Nancharaiah et 125 al., 2012). The PMS medium consisted of the following: glucose, $10.0 \mathrm{~g}$; $\mathrm{NH}_{4} \mathrm{Cl}, 0.5 \mathrm{~g}$;

$126 \mathrm{MgSO}_{4} .7 \mathrm{H}_{2} \mathrm{O}, 0.2 \mathrm{~g} ; \mathrm{KH}_{2} \mathrm{PO}_{4}, 5 \mathrm{~g} ; \mathrm{K}_{2} \mathrm{HPO}_{4} .3 \mathrm{H}_{2} \mathrm{O}, 6.55 \mathrm{~g} ; \mathrm{FeSO}_{4} .7 \mathrm{H}_{2} \mathrm{O}, 0.005$ g; peptone, $0.1 \mathrm{~g}$;

127 yeast extract, $0.1 \mathrm{~g}$; deionized water to $1 \mathrm{~L} ; \mathrm{pH}, 6.8$. The serum bottles and flasks were prepared

128 with PMS medium and autoclaved as mentioned above. [EMIM][Ac] was added to serum bottles

129 or culture flasks containing PMS medium. PMS medium without ionic liquid was used as the

130 control. The serum bottles and flasks with and without ionic liquid were inoculated with

131 Clostridium sp. and P. putida respectively and incubated as described above. All experiments 
132 were setup in triplicate serum bottles or culture flasks. The liquid samples were collected at 133 regular time intervals for monitoring growth and $\mathrm{pH}$.

1342.6 Analytical methods

135

136 137

Bacterial growth was monitored by measuring turbidity at $600 \mathrm{~nm}$ using Spectronic 20 spectrophotometer (Thermo Scientific, USA) or UV-3600 UV-Vis spectrophotometer (Shimadzu, Japan). Total gas produced by Clostridium sp. was measured using a pressure gauge connected to a syringe (Francis and Dodge, 1987). An aliquot of the culture sample was filtered through $0.45 \mu \mathrm{m}$ Millex filter and the $\mathrm{pH}$ was determined using a Beckman $350 \mathrm{pH}$ meter with a Beckman 511275-AB combination electrode. Glucose was analyzed by HPLC. The HPLC system consisted of a SCL-10A controller, a SIL-10A sample autoinjector, and a LC-10AS liquid chromatograph (Shimadzu, Japan). The HPLC system was fitted with a Bio-Rad HPX87H column (Bio-Rad labs, USA) and mobile phase $\left(0.003 \mathrm{~N} \mathrm{H}_{2} \mathrm{SO}_{4}\right)$ was pumped at flow rate of $0.7 \mathrm{ml} \mathrm{min}^{-1}$. Glucose concentration was determined using RID-6A refractive index detector (Shimadzu, Japan).

\section{Results and discussion}

\subsection{Effect of ionic liquids on Clostridium sp. growth}

The effect of water miscible imidazolium ILs with short alkyl chains on the cation (-ethyl and -methyl) and three different anion groups (acetate, diethylphosphate and dimethylphosphate) on Clostridium sp. growth indicated that the toxicity was dependant with the type of IL and its concentrationconcentration. The growth curves of Clostridium sp. could be characterized with 
152 distinct rapid exponential and extended stationary phases. Clostridium sp. grew rapidly in the

153 first $24 \mathrm{~h}$ by fermenting the glucose. This rapid exponential growth has caused a significant drop

154 in the medium $\mathrm{pH}$ to $\sim 2.8$ (Fig. 1), which has lowered the subsequent growth of Clostridium sp.

155 The growth of Clostridium sp. was higher by almost $40 \%$ in mineral salts medium in the

156 presence of lower concentrations of [EMIM][Ac]. Delay and complete inhibition in the growth

157 of Clostridium was observed in the presence of moderate and higher concentrations of

$158[\mathrm{EMIM}][\mathrm{Ac}]$, respectively. The growth of Clostridium sp. was not significantly altered in the

159 presence of [EMIM][DEP] up to $2.5 \mathrm{~g} \mathrm{~L}^{-1}$. However, the final growth was decreased in the range

160 of $9-60 \%$ in the presence of higher concentrations of [EMIM][DEP]. The growth was not

161 significantly altered by [EMIM][DMP] up to $4 \mathrm{~g} \mathrm{~L}^{-1}$. The growth of Clostridium sp. was either

162 retarded or completely inhibited at higher concentrations of [MMIM][DMP]. Overall, ionic

163 liquids used in the present study did not cause inhibition in the growth and fermentative

164 metabolism of Clostridium sp. up to $2.5 \mathrm{~g} \mathrm{~L}^{-1}$ (Fig. 1, Figs. SM-1 to SM-2). The toxicity of ionic

165 liquids was evident in either delaying the growth or causing complete growth inhibition, which is

166 seen only at higher concentrations above $>2.5 \mathrm{~g} \mathrm{~L}^{-1}$. Interestingly, [EMIM][Ac] exhibited

167 hormesis, thereby increasing the growth of Clostridium sp. at lower concentrations ranging from

1680.5 to $2.5 \mathrm{~g} \mathrm{~L}^{-1}$ (Fig. 1). At higher concentrations $\left(>2.5 \mathrm{~g} \mathrm{~L}^{-1}\right)$ of [EMIM][Ac], the growth of

169 Clostridium sp. was inhibited significantly. The growth of Clostridium sp. was completely

170 inhibited at $7.5 \mathrm{~g} \mathrm{~L}^{-1}$ and higher concentrations of [EMIM][Ac]. Whereas the other two ionic

171 liquids, $[\mathrm{EMIM}][\mathrm{DEP}]$ and $[\mathrm{MMIM}][\mathrm{DMP}]$ caused a concentration dependent inhibition in the

172 growth (Fig. SM-1) and fermentative metabolism (Figs. SM-2 and SM-3) of Clostridium sp.

1733.2 Hormetic effect of [EMIM][Ac] 
In the presence of [EMIM][Ac], the $\mathrm{pH}$ of mineral salts medium was always found to be 175 higher than that of control (Figs. 1 and 2). The $\mathrm{pH}$ of MS medium remained at 4 or higher in the 176 presence of ionic liquid that facilitated growth. Clostridium sp. growth was almost $40 \%$ higher in 177 mineral salts medium supplemented with $0.5 \mathrm{~g} \mathrm{~L}^{-1}$ of [EMIM][Ac] as compared to the growth 178 observed in mineral salts medium without ionic liquid (Fig. 2). Enhanced growth of Clostridium 179 sp. was corroborated with higher glucose utilization (Fig. SM-3) and total gas production (Fig. 180 SM-2).

The hormetic effect of [EMIM][Ac] was even observed on growth of a Gram negative 182 bacterium $P$. putida. In fact, the enhancement in the growth of $P$. putida was much higher in 183 mineral salts medium supplemented with [EMIM][Ac] (Fig. 3). The growth of $P$. putida was almost $400 \%$ higher in mineral salts medium supplemented with $0.5 \mathrm{~g} \mathrm{~L}^{-1}$ of [EMIM][Ac]. The 185 $\mathrm{pH}$ of the mineral salts medium decreased to $\sim 3.5$ within $40 \mathrm{~h}$ of inoculation and remained stable thereafter. In the presence of ionic liquid, $\mathrm{pH}$ of the medium was maintained above 6.0 until $40 \mathrm{~h}$ 187 and subsequently decreased to $\sim 3.5$. Based on the data, it was hypothesized that the hormetic 188 effect of $[\mathrm{EMIM}][\mathrm{Ac}]$ was mediated via medium $\mathrm{pH}$ regulation. This was verified by 189 determining the growth of Clostridium sp. and P. putida in phosphate buffered mineral salts 190 medium (PMS) with and without $0.5 \mathrm{~g} \mathrm{~L}^{-1}$ [EMIM][Ac]. The growth of Clostridium sp. or $P$. putida was much higher in PMS medium compared to the growth observed in MS medium. The 192 higher growth was possible because the $\mathrm{pH}$ of PMS medium maintained at $\sim 6.5$ throughout 193 growth phase. However, the growth of Clostridium sp. and P. putida were not enhanced by the 194 addition of [EMIM][Ac] to PMS medium (Figs. 4 and 5). These results were in agreement with 195 earlier findings that the stimulatory effect of [EMIM][Ac] on Clostridium sp. growth and 196 fermentative metabolism could be simulated by the addition of bicarbonate to MS medium 
197 (Nancharaiah and Francis, 2011). Moreover, [EMIM][Ac] did not show hormesis on P. putida

198 grown either in tryptic soy broth or in MS medium supplemented with a non-fermentable carbon 199 source such as acetate (data not shown).

200

201

202

203

204

205

206

207

208

209

210

211

212

213

214

215

216 217 hormetic effect of [EMIM][Ac] on Clostridium sp. or P. putida in poorly buffered medium

218

219

The toxicity of ionic liquids is associated with the type of cation, anion or the alkyl chain length of cation substituent's (Nancharaiah and Francis, 2011; Ranke et al., 2004; Nancharaiah et al., 2012; Wang et al., 2011). Certain ionic liquids, particularly those with short alkyl side chains have shown hormesis on bacteria (Ge et al., 2010; Nancharaiah and Francis 2011; Wang et al., 2011; Zhang et al., 2013a; Zhang et al., 2013b;), microalgae (Cho et al., 2007; Cho et al., 2008), IPC-81 leukemia cells (Ranke et al., 2004) and HeLa cells (Stepnowski et al., 2004). The luminescence of Vibrio qinghaiensis sp. -Q67 was induced remarkably by 1-ethyl-3methylimidazolium tetrafluoroborate (Wang et al., 2011). Ge et al. (2010) have predicted hormetic effects of ionic liquid mixtures on luciferase activity using concentration addition model. The mechanistic aspects of hormesis exhibited by ionic liquids are largely unknown. Dipeolu et al., (2009) hypothesized that N, N-dimethylethanolammonium acetate increased the growth rate of Clostridium sporogenes by either increasing the bioavailability of nutrients or by biodegradation of ionic liquid itself (Dipeolu et al., 2009). Based on the data obtained in this study, it can be concluded that the hormesis of [EMIM][Ac] on bacterial growth was is dependent on the medium buffering capacity and the type of fermentable and/or non-fermentable nature of the substrate. Bacterial growth was enhanced [EMIM][Ac] only in a poorly buffered medium that contained a fermentable substrate as the carbon source. It is apparent that the supplemented with glucose as carbon source is mostly associated with buffering action of IL in particular acetate anion group. 


\section{Conclusions}

221 Among the three water miscible ionic liquids, 1-ethyl-3-methylimidazolium acetate, 1-ethyl-3-

222 methylimidazolium diethyl phosphate and 1-methyl-3-methylimidazolium dimethylphosphate, 223 tested only [EMIM][Ac] showed remarkable hormesis on Clostridium sp. and Pseudomonas

224 putida growth. The growth was stimulated at up to $2.5 \mathrm{~g} \mathrm{~L}^{-1}$ and inhibited at $>2.5 \mathrm{~g} \mathrm{~L}^{-1}$ of 225 [EMIM][Ac]. The growth of Clostridium sp. and Pseudomonas putida increased by 0.4 and 4-

226 times, respectively in mineral salts medium supplemented with $0.5 \mathrm{~g} \mathrm{~L}^{-1}$ of [EMIM][Ac]. The

227 enhancement in bacterial growth due to [EMIM][Ac] was not evident in tryptic soy broth, 228 phosphate buffered mineral salts medium, or mineral salts medium that contained a non-

229 fermentable substrate. It is evident that the bacterial growth was enhanced only in poorly 230 buffered medium that contained a fermentable substrate as carbon source. Therefore, the 231 hormesis of [EMIM][Ac] could be alleviated by growing bacterial cultures in a phosphate 232 buffered mineral salts medium or mineral salts medium supplemented with a non-fermentable 233 substrate.Acknowledgments

234 This research was in part supported by BK-21 plus program through the National Research 235 Foundation of Korea funded by the Ministry of Education, Science, and Technology (R31 23630005 ) and by the Office of Biological and Environmental Research, Office of Science, US. 237 Department of Energy, under contract No.DE-SC00112704.

\section{$238 \quad$ References} with ionic liquids. Green Chem. 15, 550-583. 
Bubalo, M.C., Radošević, K., Redovniković, I.R., Halambek, J., Srček, V.G., 2014. A brief overview of the potential environmental hazards of ionic liquids. Ecotoxicol. Environ. Saf. 99, 1-12.

Carson, L., Chau, P.K.W., Earle, M.J., Gilea, M.A., Gilmore, B.F., Gorman, S.P., McCann, M.T., Seddon, K.R., 2009. Anti-biofilm activities of 1-alkyl-3-methylimidazolium chloride ionic liquids. Green Chem. 11, 492-497.

Cho, C.W., Pham, T.P., Jeon, Y.C., Vijayaraghavan, K., Choe, W.S., Yun, Y.S., 2007. Toxicity of imidazolium salt with anion bromide to a phytoplankton Selenastrum capricornutum: effect of alkyl-chain length. Chemosphere 69, 1003-1007.

Cho, C.W., Jeon, Y.C., Pham, T.P., Vijayaraghavan, K., Yun, Y.S., 2008. The ecotoxicity of ionic liquids and traditional organic solvents on microalga Selenastrum capricornutum. Ecotoxicol. Environ. Saf. 71, 166-171.

Datta, S., Holmes, B., Park, J.I., Chen, Z., Dibble, D.C., Hadi, M., Blanch, H.W., Simmons, B.A., Sapra, R., 2010. Ionic liquid tolerant hyperthermophilic cellulases for biomass pretreatment and hydrolysis. Green Chem. 12, 338-345.

Davies, J., Spiegelman, G.B., Yim, G., 2006. The world of subinhibitory antibiotic concentrations. Curr. Opin. Microbiol. 9, 445-453.

Dipeolu, O., Green, E., Stephens, G., 2009. Effects of water-miscible ionic liquids on cell growth and nitro reduction using Clostridium sporogenes. Green Chem. 11, 397-401. 
Earle, M.J., Seddon, K.R., 2000. Ionic liquids. green solvents for the future. Pure Appl. Chem. 72, 1391-1398.

Francis, A.J., Dodge, C.J., 1987. Effects of lead oxide and iron on glucose fermentation of Clostridium sp. Arch. Environ. Contam. Toxicol. 16, 491-497.

Ge, H.L., Liu, S.S., Zhu, X.W., Liu, H.L., Wang, L.J., 2010. Predicting hormetic effects of ionic liquid mixtures on luciferase activity using the concentration addition model. Environ. Sci. Technol. 45, 1623-1629.

Kuhlmann, E., Himmler, S., Giebelhaus, H., Wasserscheid, P., 2007. Imidazolium dialkylphosphates - a class of versatile, halogen-free and hydrolytically stable ionic liquids. Green Chem. 9, 233-242.

Nancharaiah, Y.V., Gupta, A., Dodge, C.J., Francis, A.J., 2012. Effect of exogenous electron shuttles on growth and fermentative metabolism in Clostridium sp. BC1. Bioresour. Technol. 108, 195-299.

Nancharaiah, Y.V., Francis, A.J., 2011. Alkyl-imidazolium ionic liquids affect the growth and fermentative metabolism of Clostridium sp. Bioresour. Technol. 102, 6573-658.

Nancharaiah, Y.V., Joshi, H.M., Hausner, M., Venugopalan, V.P., 2008. Bioaugmentation of aerobic microbial granules with Pseudomonas putida carrying TOL plasmid. Chemosphere 71, 30-35. 
278 Nancharaiah, Y.V., Kiran Kumar Reddy, G., Lalithamanasa, P., Venugopalan, V.P., 2012. The

279 ionic liquid 1-alkyl-3-methylimidazolium demonstrates comparable antimicrobial and antibiofilm behavior to a cationic surfactant. Biofouling 28, 1141-1149.

Nancharaiah, Y.V., Wattiau, P., Wuertz, S., Bathe, S., Mohan, S.V., Wilderer, P.A., Hausner, M., 2003. Dual labeling of Pseudomonas putida with fluorescent proteins for in situ monitoring of conjugal transfer of the TOL plasmid. Appl. Environ. Microb. 69, 48464852.

Passos, H., Freire, M.G., Coutinho, J.A., 2014. Ionic liquid solutions as extractive solvents for value-added compounds from biomass. Green Chem. 16, 4786-4815.

Patel, D.D., Lee, J-M., 2012. Applications of ionic liquids. Chem. Rec. 12, 329-355.

Pham, T.P., Cho, C-W., Yun, Y-S., 2010. Environmental fate and toxicity of ionic liquids: a review. Water Res. 44, 352-372.

Plechkova, N.V., Seddon, K.R., 2008. Applications of ionic liquids in the chemical industry. Chem. Soc. Rev. 37, 123-150.

Ranke, J., Mölter, K., Stock, F., Bottin-Weber, U., Poczobutt, J., Hoffmann, J., Ondruschka, B., Filser, J., Jastorff, B., 2004. Biological effects of imidazolium ionic liquids with varying chain lengths in acute Vibrio fischeri and WST-1 cell viability assays. Ecotoxicol. Environ. Saf. 58, 396-404. 
Stepnowski, P., Składanowski, A.C., Ludwiczak, A., Łaczynřska, E., 2004. Evaluating the cytotoxicity of ionic liquids using human cell line HeLa. Hum. Exp. Toxicol. 23, 513517.

Swatloski, R.P., Spear, S.K., Holbrey, J.D., Rogers, R.D., 2002. Dissolution of cellulose with ionic liquids. J. Am. Chem. Soc. 124, 4974-4975.

Stepnowski, P., Skladanowski, A.G., Ludwiczak, A., Laczynařska, E., 2004. Evaluating cytotoxicity of ionic liquids using human cell line HeLa. Hum. Exp. Toxicol. 23, 513517.

Wang, L.J., Liu, S.S., Yuan, J., Liu, H.L., 2011. Remarkable hormesis induced by 1-alkyl-3methyl imidazolium tetrafluoroborate on Vibrio qinghaiensis sp.-Q67. Chemosphere 84, $1440-1445$.

Vitz, J., Erdmenger, T., Haensch, C., Schubert, U.S., 2009. Extended dissolution studies of cellulose in imidazolium based ionic liquids. Green Chem. 11, 417-424.

Wang, H., Malhotra, S.V., Francis, A.J., 2011. Toxicity of various anions associated with methoxyethyl methyl imidazolium-based ionic liquids on Clostridium sp. Chemosphere 82, 1597-1603.

Zavrel, M., Bross, D., Funke, M., Büchs, J., Spiess, A.C., 2009. High-throughput screening for ionic liquids dissolving (ligno-) cellulose. Bioresour. Technol. 100, 2580-2587. 
314 Zhang, J., Liu, S-S., Yu, Z-Y., Zhang, J., 2013a. Time-dependent hormetic effects of 1-alkyl-3methylimidazolium bromide on Vibrio qinghaiensis sp.-Q67: luminescence, redox reactants and antioxidants. Chemosphere 91, 462-467.

317 Zhang, J., Liu, S-S., Yu, Z-Y., Liu, H-L., Zhang, J., 2013b. The time-dependent hormetic effects 318 of 1-alkyl-3-methylimidazolium chloride and their mixtures on Vibrio qinghaiensis sp.Q67. J. Hazar. Mater. 258-259, 70-76. 
322 Table 1. The structure of ionic liquids used in the present study.

\begin{tabular}{lll}
\hline IL name & Abbreviation \\
$\begin{array}{l}\text { 1-ethyl-3-methylimidazolium } \\
\text { acetate }\end{array}$ & {$[\mathrm{EMIM}][\mathrm{Ac}]$} \\
$\begin{array}{l}\text { 1-ethyl-3-methylimidazolium } \\
\text { diethylphosphate } \\
\text { 1,3-dimethylimidazolium } \\
\text { dimethylphosphate }\end{array}$ & {$[\mathrm{EMIM}][\mathrm{DEP}]$} \\
{$[\mathrm{MMIM}][\mathrm{DM}$}
\end{tabular}

323

324

325 
$327 \quad$ Figure legends

328 Figure 1. Growth (A) and medium $\mathrm{pH}$ (B) of Clostridium sp. BC1 in mineral salts medium 329 supplemented with different concentrations of 1-ethyl-3-methylimidazolium acetate 330 ([EMIM][Ac]). Growth measured at $96 \mathrm{~h}$ of inoculation is shown. Symbols and error bars 331 represent averages and standard deviations from triplicate experiments.

332 Figure 2. Clostridium sp. BC1 growth (A) and medium $\mathrm{pH}(\mathrm{B})$ in mineral salts (MS) medium 333 (control), and MS medium amended with $0.5 \mathrm{~g} \mathrm{~L}^{-1}$ [EMIM][Ac]. Symbols and error bars 334 represent averages and standard deviations from triplicate experiments.

335 Figure 3. Clostridium sp. BC1 growth (A) and medium $\mathrm{pH}(\mathrm{B})$ in phosphate buffered mineral 336 salts (PMS) medium (control), and PMS medium amended with $0.5 \mathrm{~g} \mathrm{~L}^{-1}$ [EMIM][Ac]. Symbols 337 and error bars represent averages and standard deviations from triplicate experiments.

Figure 45. P. putida growth (A) and medium $\mathrm{pH}$ (B) in mineral salts (MS) medium (control), MS medium amended with $0.5 \mathrm{~g} \mathrm{~L}^{-1}$ [EMIM][Ac]. Symbols and error bars represent averages and 340 standard deviations from triplicate experiments.

341 Figure 5. P. putida growth (A) and medium $\mathrm{pH}$ (B) in phosphate-buffered mineral salts (PMS) 342 medium (control), PMS medium amended with $0.5 \mathrm{~g} \mathrm{~L}^{-1}$ [EMIM][Ac]. Symbols and error bars 343 represent averages and standard deviations from triplicate experiments. 

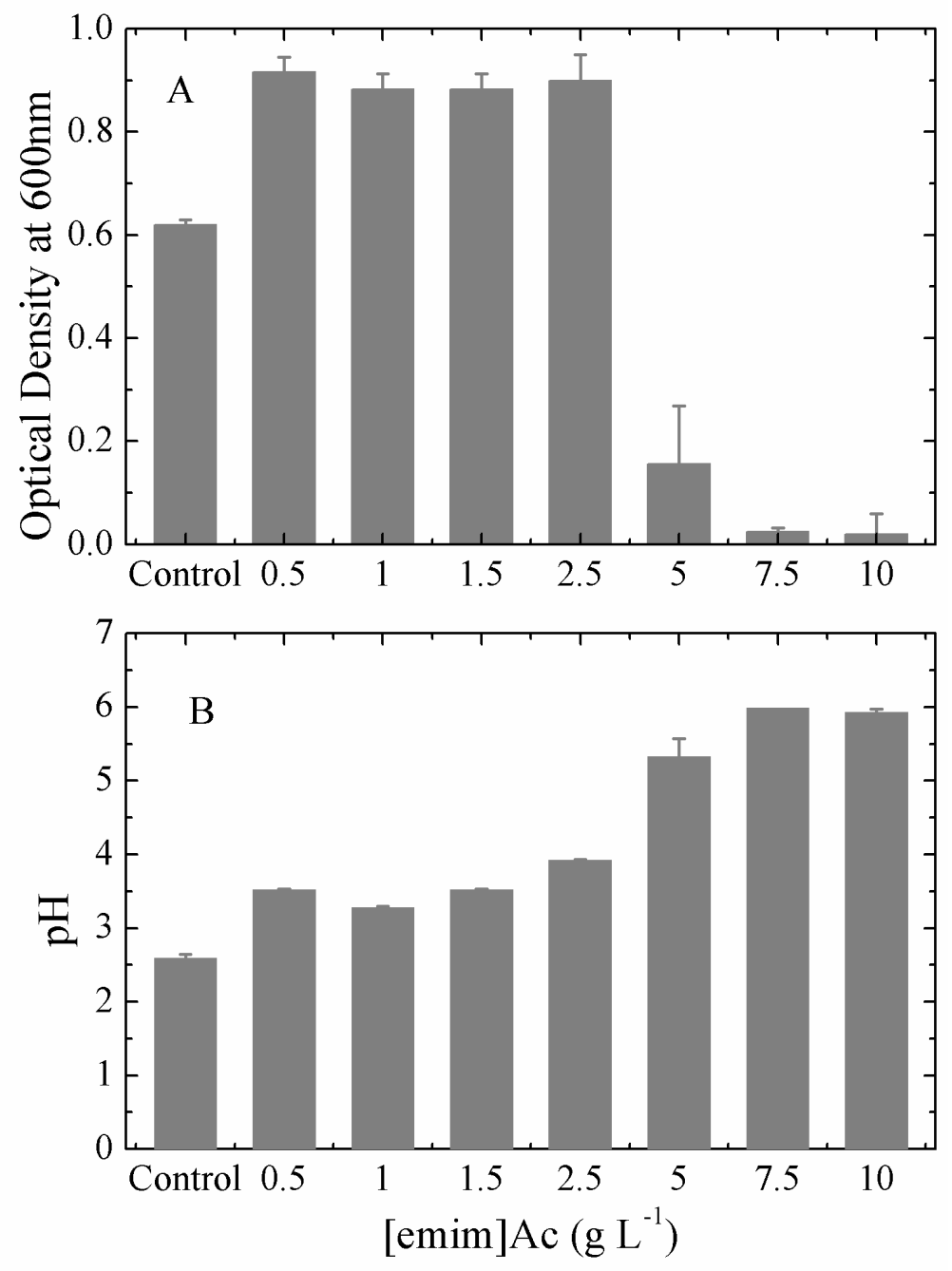

347 Figure 1. Growth (A) and medium $\mathrm{pH}(\mathrm{B})$ of Clostridium $\mathrm{sp}$. BC1 in mineral salts medium 348 supplemented with different concentrations of 1-ethyl-3-methylimidazolium acetate 349 ([EMIM][Ac]). Growth was measured at $96 \mathrm{~h}$ of inoculation is shown. Symbols and error bars 350 represent averages and standard deviations from triplicate experiments. 

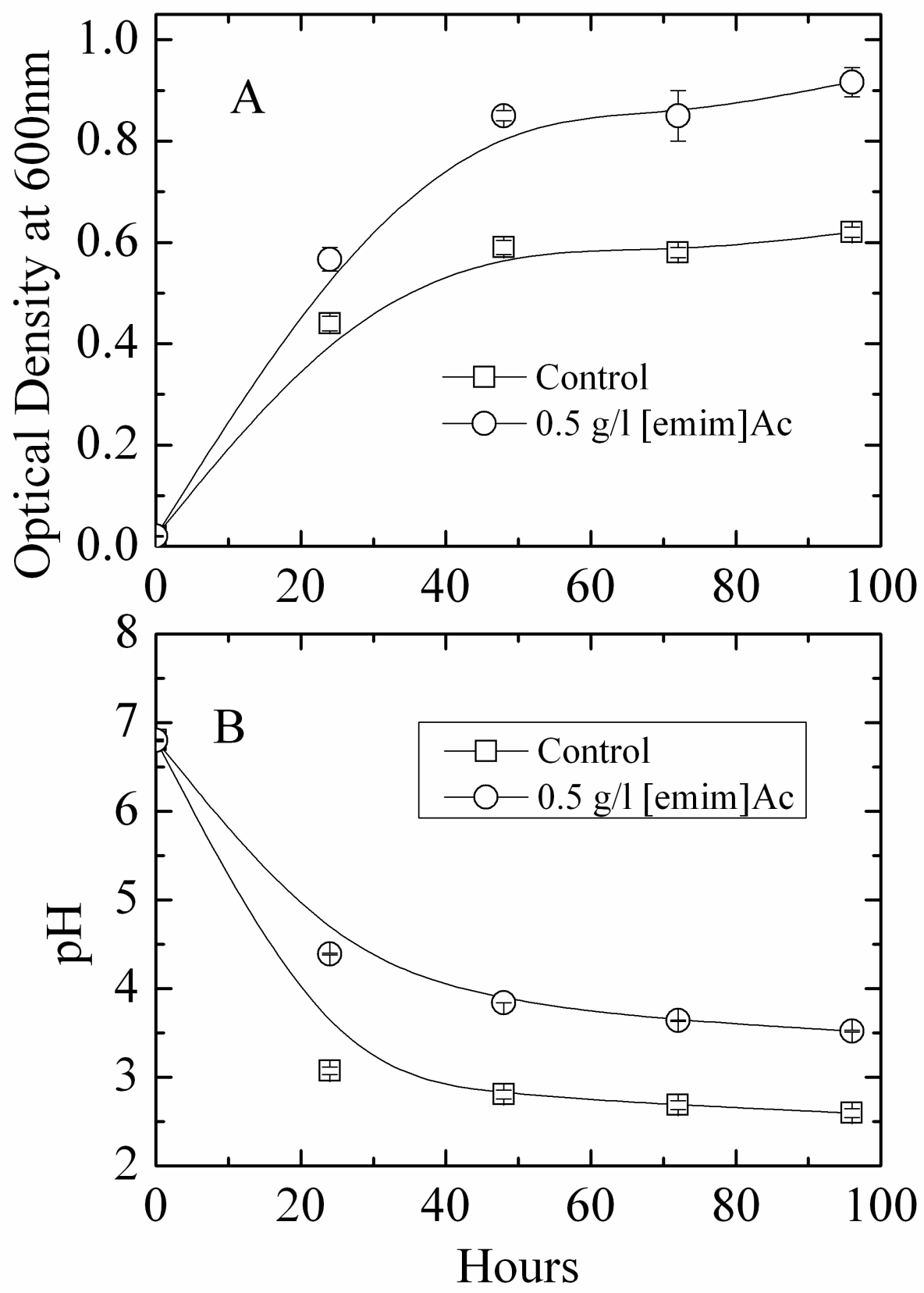

Figure 2. Clostridium sp. BC1 growth (A) and medium $\mathrm{pH}(\mathrm{B})$ in mineral salts (MS) medium (control), and MS medium amended with $0.5 \mathrm{~g} \mathrm{~L}^{-1}$ [EMIM][Ac]. Symbols and error bars represent averages and standard deviations from triplicate experiments. 

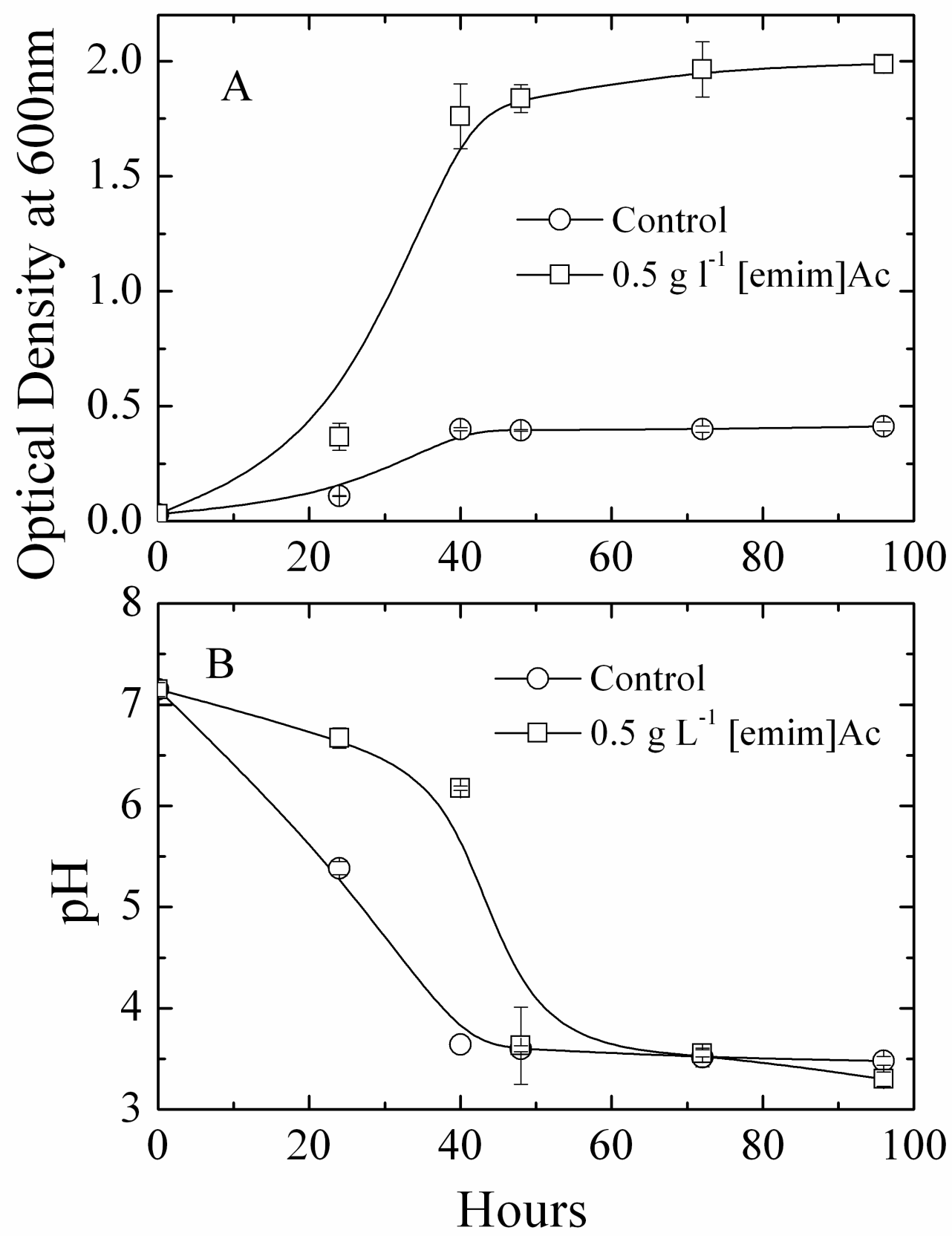

Figure 3. Clostridium sp. $\mathrm{BC} 1$ growth (A) and medium $\mathrm{pH}(\mathrm{B})$ in phosphate buffered mineral salts (PMS) medium (control), and PMS medium amended with $0.5 \mathrm{~g} \mathrm{~L}^{-1}$ [EMIM][Ac]. Symbols and error bars represent averages and standard deviations from triplicate experiments. 

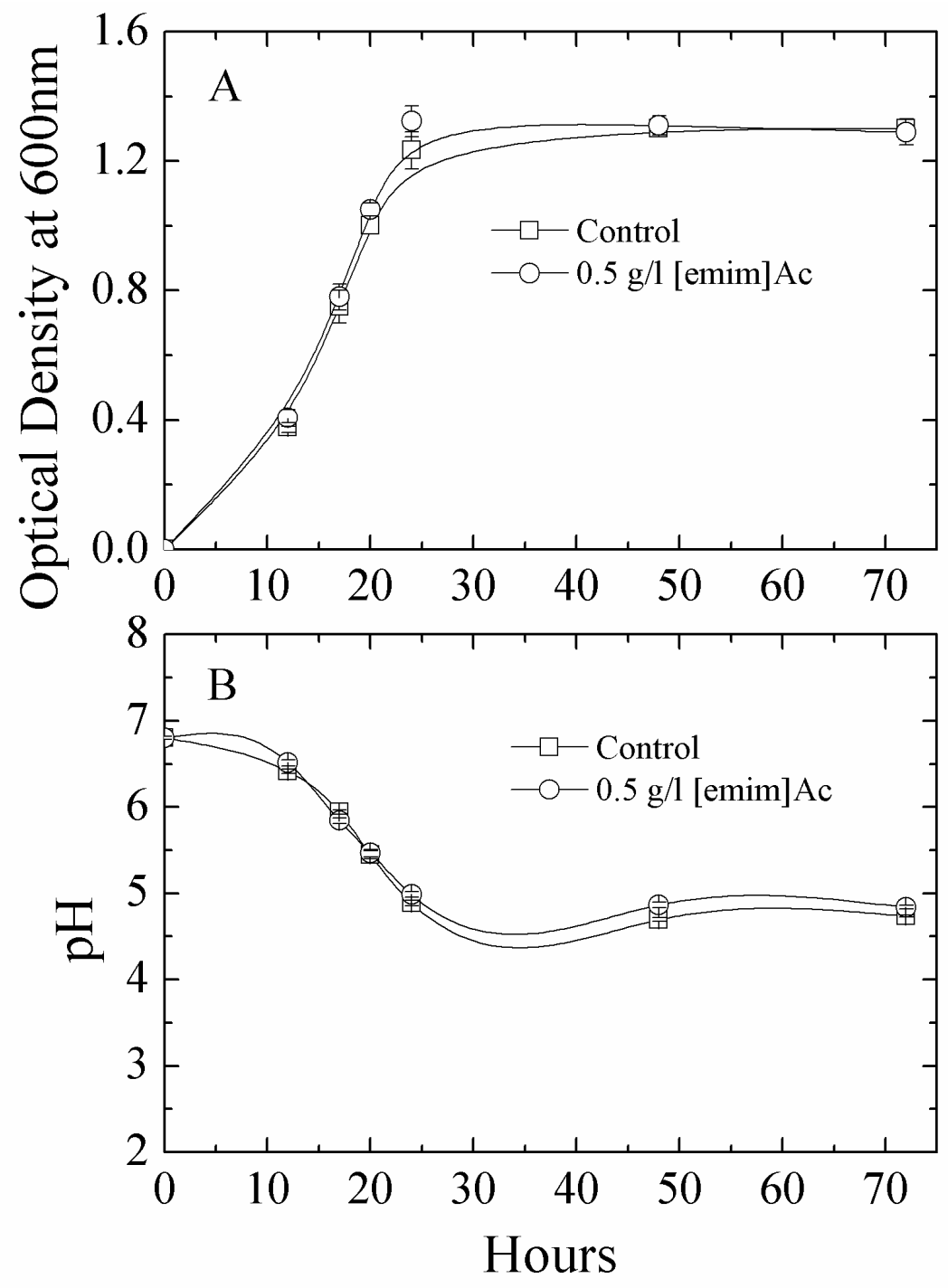

371

372
Figure 4. P. putida growth (A) and medium $\mathrm{pH}(\mathrm{B})$ in mineral salts (MS) medium (control), MS medium amended with $0.5 \mathrm{~g} \mathrm{~L}^{-1}$ [EMIM][Ac]. Symbols and error bars represent averages and standard deviations from triplicate experiments. 

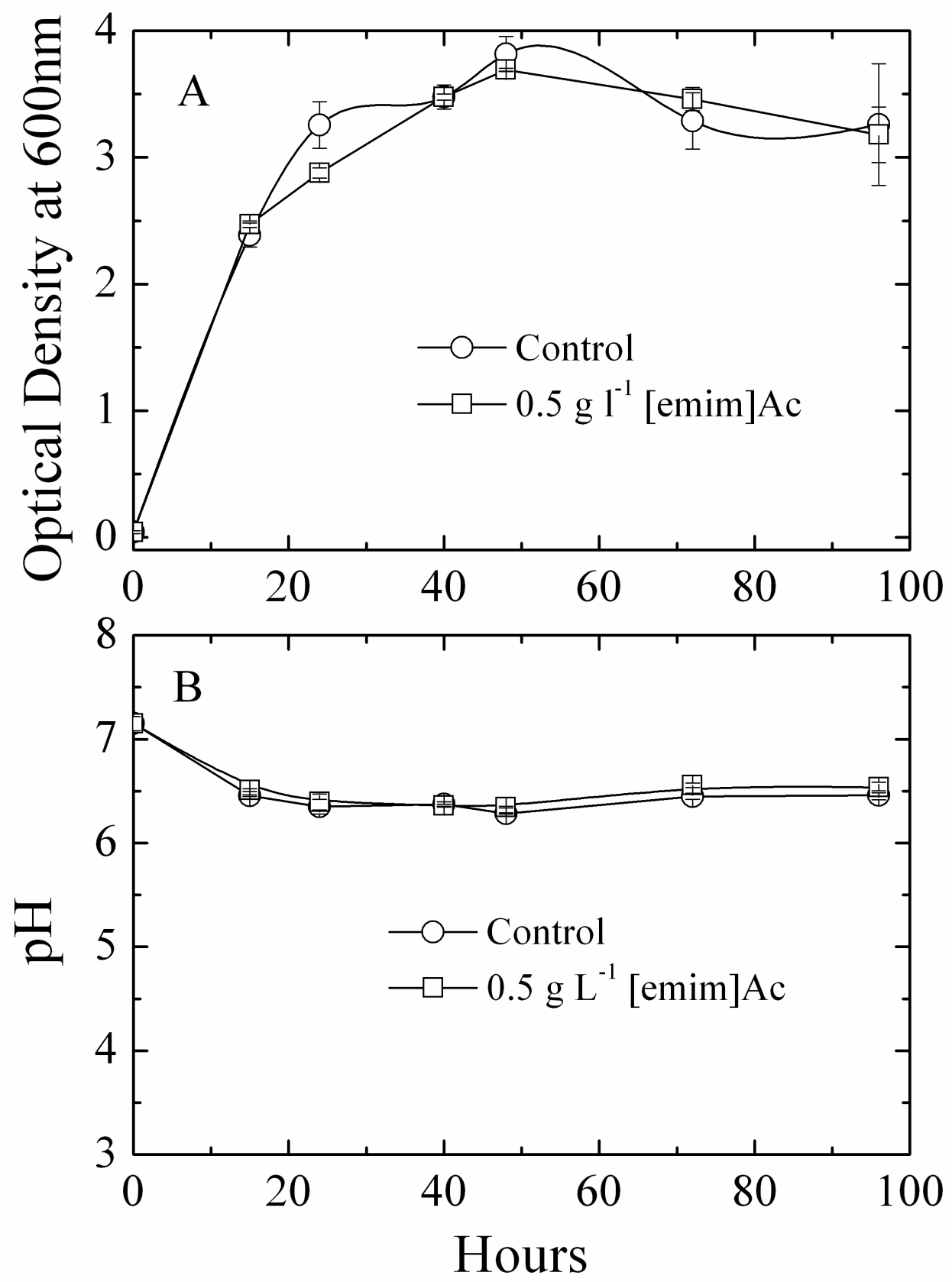

373

374 Figure 5. P. putida growth (A) and medium $\mathrm{pH}(\mathrm{B})$ in phosphate-buffered mineral salts (PMS) 375 medium (control), PMS medium amended with $0.5 \mathrm{~g} \mathrm{~L}^{-1}$ [EMIM][Ac]. Symbols and error bars 376 represent averages and standard deviations from triplicate experiments. 